\title{
ASO Visual Abstract: Multidisciplinary Review of Intraductal Papilloma of the Breast Can Identify Patients Who May Omit Surgical Excision
}

\author{
Shahrzad Abbassi-Rahbar, MD ${ }^{1}$, Stephen Sack, BA ${ }^{2}$, Kelsey E. Larson, MD ${ }^{1}$, Jamie L. Wagner, DO ${ }^{1}$, \\ Lyndsey J. Kilgore, MD ${ }^{1}$, Christa R. Balanoff, MD, MHPE ${ }^{1}$, Onalisa D. Winblad, $\mathrm{MD}^{3}$, and \\ Amanda L. Amin, MD, MS \\ ${ }^{1}$ Department of Surgery, The University of Kansas Health System, Kansas City, KS; ${ }^{2}$ University of Kansas Medical \\ Center, Kansas City, KS; ${ }^{3}$ Department of Radiology, The University of Kansas Health System, Kansas City, KS
}

The aim of our study was to define contemporary management recommendations regarding who would benefit from surgical excision of intraductal papilloma (IDP) (https://doi.org/10.1245/s10434-021-10520-1).

A prospective database from a single institution identified patients with IDP on percutaneous biopsy from 02/2015 to 09/2020. Categorical patient demographic, biopsy, and pathologic variables were analyzed using Fisher's exact test and continuous demographic and imaging variables were analyzed using the Mann-Whitney U test.

IDP was present in 416 biopsies, at a median age of 56. The median size was $0.9 \mathrm{~cm}$, and the majority had greater than $50 \%$ of the target excised by biopsy. Surgical excision was performed for 124/416 biopsies (29.8\%). Upgrade to malignancy was identified in 14 (11.3\%): 8 to ductal carcinoma in situ (DCIS) and 6 to invasive cancer. Upgrade was significantly associated with concurrent ipsilateral breast cancer $(p=0.027)$, larger imaging size $(p=0.045),<50 \%$ excised with biopsy $(p=0.02)$, and atypia involving IDP $(p=0.045)$. Age, clinical presentation, and concurrent contralateral cancer were not significantly associated with upgrade. Lowest upgrade risk $(0 \%)$ was in pure IDP $\leq 1 \mathrm{~cm}$ with $>50 \%$ removed by biopsy. Of 401 biopsies that either did not upgrade or undergo excision, 7 (1.7\%) developed subsequent breast cancer over a median follow-up of 23.5 months (IQR 11.41), none at the IDP site.

After multidisciplinary review, the management of IDP can be stratified into low and high-risk for upgrade groups, using key criteria. The low-risk group may omit surgical excision, as those patients have $0 \%$ risk of upgrade over the limited short-term follow up.

Supplementary Information The online version contains supplementary material available at https://doi.org/10.1245/s10434021-10624-8.

DISCLOSURES The authors declare that there is no conflict of interest.

Publisher's Note Springer Nature remains neutral with regard to jurisdictional claims in published maps and institutional affiliations.

(C) Society of Surgical Oncology 2021

Published Online: 1 September 2021

A. L. Amin, MD, MS

e-mail: aamin624@gmail.com 